\title{
Formative period in the $x$-ray-induced photodissociation of organic molecules
}

\author{
E. Kukk $\odot,{ }^{1}$ H. Fukuzawa $\odot,{ }^{2,3}$ J. Niskanen, ${ }^{1}$ K. Nagaya, ${ }^{4}$ K. Kooser, ${ }^{1,5}$ D. You, ${ }^{2}$ J. Peschel, ${ }^{6}$ S. Maclot $\odot,{ }^{6}$ A. Niozu, ${ }^{4}$ \\ S. Saito, ${ }^{2}$ Y. Luo, ${ }^{2}$ E. Pelimanni $\odot,{ }^{7}$ E. Itälä, ${ }^{1}$ J. D. Bozek, ${ }^{8}$ T. Takanashi, ${ }^{2}$ M. Berholts $\odot,{ }^{5,8}$ P. Johnsson $\odot,{ }^{6}$ and K. Ueda ${ }^{2,3}$ \\ ${ }^{1}$ Department of Physics and Astronomy, University of Turku, FI-20014 Turku, Finland \\ ${ }^{2}$ Institute of Multidisciplinary Research for Advanced Materials, Tohoku University, Sendai 980-8577, Japan \\ ${ }^{3}$ RIKEN SPring-8 Center, Kouto 1-1-1, Sayo, Hyogo 679-5148, Japan \\ ${ }^{4}$ Department of Physics, Kyoto University, Kyoto 606-8502, Japan \\ ${ }^{5}$ Institute of Physics, University of Tartu, Wilhelm Ostwaldi 1, EE-50411 Tartu, Estonia \\ ${ }^{6}$ Department of Physics, Lund University, SE-22100 Lund, Sweden \\ ${ }^{7}$ Nano and Molecular Systems Research Unit, Faculty of Science, University of Oulu, FI-90014 Oulu, Finland \\ ${ }^{8}$ Synchrotron SOLEIL, L'Orme des Merisiers, Saint-Aubin, BP 48, FR-91192 Gif-sur-Yvette Cedex, France
}

(Received 14 July 2020; revised 10 December 2020; accepted 7 December 2020; published 9 March 2021)

\begin{abstract}
Absorption of x-ray photons by atomic inner shells of light-element organics and biomolecules often leads to formation of dicationic electronic states and to molecular fragmentation. We investigated the x-ray-induced dissociation landscape of a representative medium-sized organic molecule, thiophene, by femtosecond x-ray pulses from the Super Photon Ring-8 GeV (SPring-8) Angstrom Compact Free-Electron Laser (SACLA). Holes, created in the sulfur $2 p$ orbital by photoemission, were filled by the Auger process that created dicationic molecular states within a broad range of internal energies-a starting point particular to x-ray-induced dynamics. The evolution of the ionized molecules was monitored by a pump-probe experiment using a near-infrared $(800 \mathrm{~nm})$ laser pulse. Ion-ion coincidence and ion momentum analysis reveals enhanced yields of ionic fragments from multibody breakup of the ring, attributed to additional ionization of the highly excited fraction of the dicationic parent molecular states. The transient nature of the enhancement and its decay with about a 160-fs time constant indicate formation of an open-ring parent geometry and the statistical survival time of the parent species before the dissociation events. By probing specific Auger final states of transient, highly excited nature by near-infrared light, we demonstrate how pump-probe signatures can be related to the key features in dynamics during the early period of the x-ray-induced damage of organic molecules and biomolecules.
\end{abstract}

DOI: 10.1103/PhysRevResearch.3.013221

\section{INTRODUCTION}

Absorption of light by molecules leads to a rich variety of photochemistry and photophysics. These processes can be initiated by nonionizing absorption of ultraviolet (UV) light, such as the ubiquitous and important $\pi \rightarrow \pi^{*}$ transitions in organic molecules [1,2], by valence ionization with vacuum ultraviolet (VUV) radiation, or by deeper atomic inner-shell ionization using $\mathrm{x}$ rays. The latter originate from either natural or artificial sources as in medical imaging and radiotherapy. When $\mathrm{x}$ rays interact with biomolecules and organic molecules consisting mainly of light elements, they remove electrons from the atomic inner shells. In a few femtoseconds, the electronic structure transitions to a lower-energy state via a radiationless Auger process, in which the inner-shell vacancy is filled, another electron is emitted, and two outer-shell vacancies are created. The resulting doubly ionized molecules most likely break up, releasing neutral and charged fragments

Published by the American Physical Society under the terms of the Creative Commons Attribution 4.0 International license. Further distribution of this work must maintain attribution to the author(s) and the published article's title, journal citation, and DOI. into the surroundings, spreading radiation damage. In this paper, we focus on the early period following inner-shell (core) ionization by $\mathrm{x}$ rays of the organic molecule thiophene. This aromatic nine-atom molecule is in the size range of the building blocks of large biomolecules and was chosen for its feature-rich dissociation landscape representing diverse molecular dynamics. Thiophene is also the basis of polyand oligothiophenes that are promising organic materials for technological applications [3].

Fundamental interest in the early photoinduced dynamics in small quantum systems arises since it largely defines the dissociation landscape-the various fragmentation pathways and the branching ratios between them. Theoretical treatment of the evolution during the first tens and hundreds of femtoseconds following photoionization is challenging due to strongly coupled and concurrent electron and nuclear dynamics in the system [4,5]. Furthermore, the properties of dicationic states and their dynamics, in which Coulomb repulsion and charge separation play an important role [6], are very different from the dynamics of cations, prevalently created by VUV and extreme ultraviolet (XUV) absorption.

The emergence of short-pulse ionizing light sources such as free-electron lasers (FELs) and high-order-harmonic generation (HHG) sources has revolutionized femtochemistry 
and related physics, allowing us to follow photodynamics in real time [7]. Various pump-probe techniques combining two light pulses with either the same or even vastly different wavelengths provide glimpses into the evolution of quantum systems ranging from single atoms to molecules and to clusters of thousands of atoms [8-10]. A popular approach to study ultrafast molecular dynamics is realized by ionizing the valence or inner-valence orbitals by VUV or XUV light and then probing the development of the cationic states by pulses of longer wavelength such as near-infrared (NIR) light [11]. NIR radiation has been shown to successfully probe VUVor XUV-induced photodynamics, from the classic experiment of the acetylene isomerization [11-14] to the much more complex processes in thymidine [15]. In the x-ray regime, experimentalists often take advantage of the extreme intensity of the short FEL pulses, creating highly charged exotic states by multiphoton absorption that undergo destructive Coulomb explosions [16-19,19-24] — a dynamics again very different from the evolution of a low-charge state. In contrast, in this paper we used strongly attenuated short (60 fs), soft x-ray pulses produced by a FEL to create a core-ionized state. We demonstrate how the subsequent probing of the photodynamics by NIR pulses reveals the early evolution of a much gentler but at the same time much more common and practically relevant event in organic molecules and biomolecules: single-photon core ionization followed by Auger decay.

Time-resolved probing of the early period of the x-rayinduced dissociation landscape allows us to address some key factors defining the final dissociation landscape and test the underlying assumptions in theoretical modeling of the photodynamics. It can reveal, for example, whether the molecules embark on a certain pathway immediately after the Auger decay of the parent dicationic species, or have a certain "survival" time. With the increasing size of the system, answering such questions by detailed mapping of the potential-energy surfaces of the many states involved becomes infeasible. However, simulation results of molecular dynamics of thiophene dications [25] suggest another description: The system embarks on a chosen dissociation pathway once the interference of the many vibrational modes creates a favorable local concentration of energy for bond breaking. Depending on the average energy of the vibrational modes and the initial conditions such as the charge state, such an event could occur with a significant delay. In time-resolved experiments with highly charged small quantum systems, survival of the intact system for any significant time is unlikely as a violent Coulomb explosion immediately follows the intense FEL pulse. However, in a doubly charged system even with very high excess internal energy - the most common starting point of x-ray-induced molecular dynamics of organic molecules and biomolecules - the parent survival time might be a very significant factor in the formative period of the dissociation.

A characteristic feature of the early dynamics is the rapidly changing electronic structure, the details of which are sensitive to how the initial state is created. Auger decay can leave the molecule in highly excited electronic states tens of $\mathrm{eV}$ above the lowest dicationic level. Recent research in biomolecular building-block molecules (in the size range of thiophene) has found that electronic relaxation of excited states through conical intersections is much more common

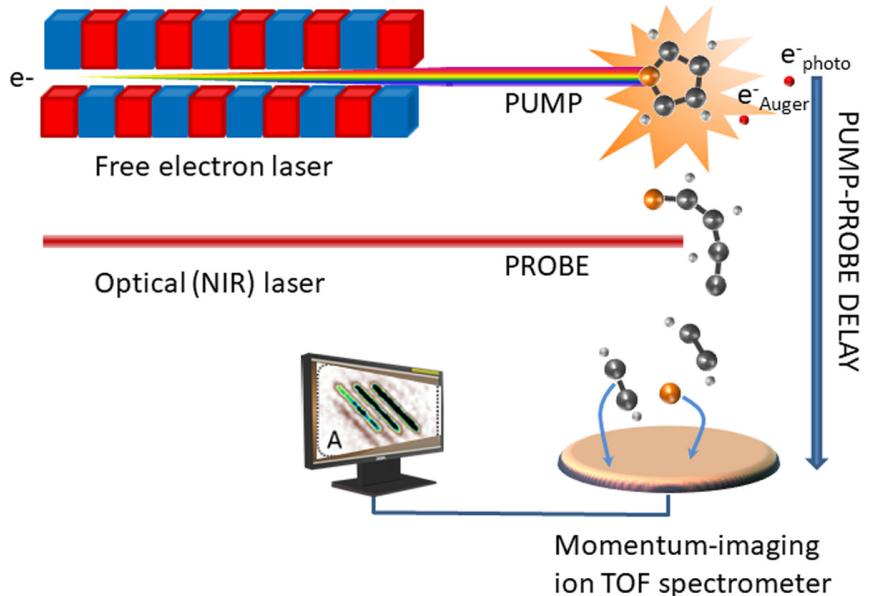

FIG. 1. Schematic of the experiment.

than once thought $[13,15,26]$. How quickly do such processes happen, how do they affect the dissociation landscape, and what are the implications for theoretical modeling of the molecular dynamics? In a common approximation the electronic relaxation to the dicationic ground state is assumed to be so fast that all electronic energy is converted to heat that then drives the dynamics on the dicationic ground-state potential-energy surface. However, if this energy conversion happens on a timescale comparable to or even longer than the time period in which the dissociation pathways are chosen, a more rigorous theoretical treatment of excited electronic states could be required.

Last but not least, this study introduces a novel approach for obtaining experimental time-dependent information on the very complex electron-nuclear dynamics of the very commonplace dicationic molecular states, created by x-ray absorption after the initial Auger relaxation of the core hole. We employ the near-infrared laser probe pulse to follow up on the x-ray ionization event. Such a probe would not be sensitive to these Auger final states but for the recently discovered (single photon) laser-enabled Auger decay (LEAD) effect [27]. We demonstrate how the LEAD-activated NIR-probe technique works and creates strong time-dependent signatures of the state of the system-not only a medium-sized organic molecule as in the present case but also a biosystem undergoing x-ray absorption and subsequent radiation damage in radiotherapy, for example.

\section{EXPERIMENTAL SETUP}

The experiments were carried out at Beamline 1 [28] of the Super Photon Ring-8 GeV (SPring-8) Angstrom Compact Free-Electron Laser (SACLA) FEL facility [29] operating at a repetition rate of $60 \mathrm{~Hz}$, with the approval of the Japan Synchrotron Radiation Research Institute (JASRI) (Proposal No. 2018B8014). The arrangement of the experiment is depicted in Fig. 1. The sample [thiophene ( $\geqslant 98.0 \%$ purity), purchased from Nacalai Tesque] was evaporated at room temperature from liquid phase without further purification and introduced into the focal spot of the FEL and NIR beams through a pulsed valve. 
The duration of the FEL pulse was estimated to be about $60 \mathrm{fs}$. The photon energy of $180 \mathrm{eV}$ was selected as the third harmonic of the undulator radiation, tuned to the maximum cross section of the sulfur $2 p$ photoabsorption. The first harmonic was removed by a $0.1-\mu \mathrm{m}$ Sn filter with a $0.016 \%$ transmission at $60 \mathrm{eV}$ and about 4400-times-higher transmission at $180 \mathrm{eV}$. The fraction of the total FEL-pulse energy that contained the third harmonic is $0.15-0.6 \%$. The removal of the first harmonic thus greatly reduced the pulse intensity, bringing the experiment to the intended single-photon inner-shell absorption regime. The 800-nm NIR pulses were generated by a Ti:sapphire laser and arrived at the interaction point with an adjustable time delay (positive or negative) relative to the arrival of the FEL pulse. The pulse duration of the NIR laser was about $30 \mathrm{fs}$ [30], the chosen attenuated pulse energy was $150 \mu \mathrm{J}$ (FWHM), and the power density in the waist of the beam was about $6 \times 10^{13} \mathrm{~W} / \mathrm{cm}^{2}$. The NIR-pulse intensity for this experiment was chosen to minimize direct multiphoton ionization of the thiophene molecules by NIR radiation only, while maintaining a sufficient cross section for the NIR pulses to interact with the FEL-ionized target.

The beamline is equipped with a cross-correlation pulse arrival time monitor [30]. Recording the measured FEL-toNIR delays for each pump-probe pulse allows us to take into account the timing jitter and improve the instrumental delaytime resolution by removing the jitter component, which in the present experiment had a standard deviation of $55 \mathrm{fs}$. The narrowest recorded broadening was $w=35(7)$ fs at the rising edge of $\mathrm{Xe}^{2+}$ (from Xe clusters) yield, fitted by Eq. (3).

Positive ions were detected by a multicoincidence momentum-imaging ion time-of-flight (TOF) spectrometer equipped with a Roentdek HEX120 position-sensitive ion detector [31]. Multiple ions could be detected per pump-probe event, and for each ion, its TOF and the hit coordinates on the 120-mm-diameter detector were recorded. From these variables, the mass-to-charge values of the ions as well as their momentum vectors were derived.

\section{RESULTS}

\section{A. Dissociation landscape of the thiophene dication after core ionization}

Before presenting our experimental findings and their interpretation in light of the above questions, we review briefly the dissociation landscape of thiophene dications and the predictions of theoretical simulations.

Interleaved with time-resolved measurements, the dissociation products of the thiophene dication were recorded in a single-pulse "FEL-only" experiment at a photon energy of $180 \mathrm{eV}$. The raw data consist of fragment ion TOF values and reconstructed momentum vectors for all ions, collected for each FEL pulse. The FEL-only experiment provided reference values for the analysis of pump-probe data.

A schematic representation of the electron and nuclear dynamics is presented in Fig. 2. In the absence of the second, NIR pulse, the molecule evolves mostly along the various dicationic potential-energy surfaces guiding it to any of the possible dissociation pathways.

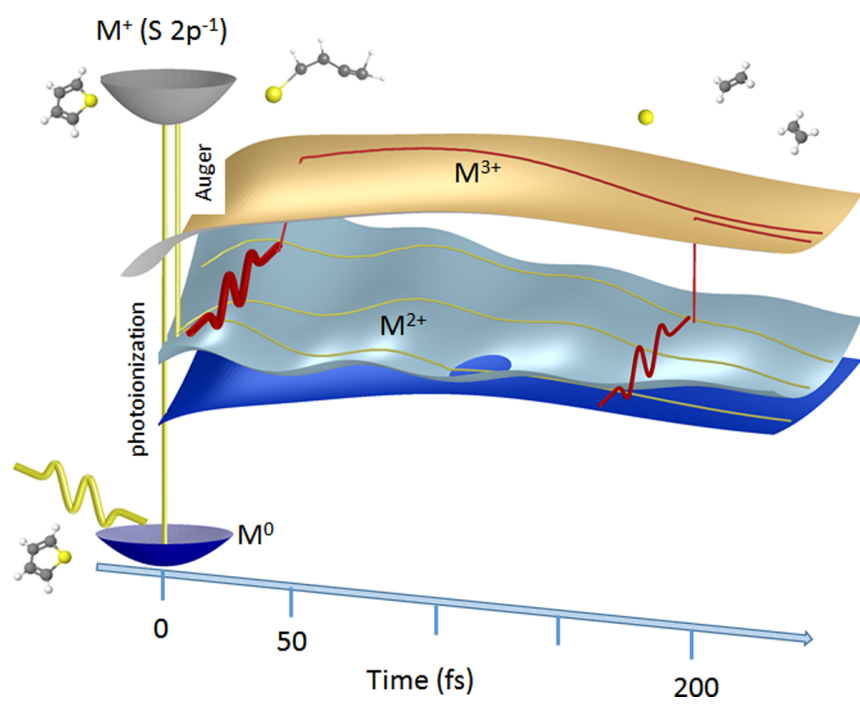

FIG. 2. Schematic depiction of the processes involved in the $x$ ray-pump-NIR-probe experiment of thiophene following S $2 p$ core ionization. The x-ray-induced transitions and evolution of the system on the dicationic $\left(\mathrm{M}^{2+}\right)$ potential-energy surfaces are marked in yellow, while the absorption of the NIR radiation lifting the dynamics onto the $\left(\mathrm{M}^{3+}\right)$ surfaces is shown in red. The evolution of molecular geometry is shown at a few points in time. The actual number of the surfaces is much larger than the few sketched.

The FEL-only measurement can be summarized in the form of a two-dimensional histogram, the photoion-photoion coincidence (PIPICO) map as shown in Fig. 3, plotting all possible ion pairs that can be formed from the ions per each

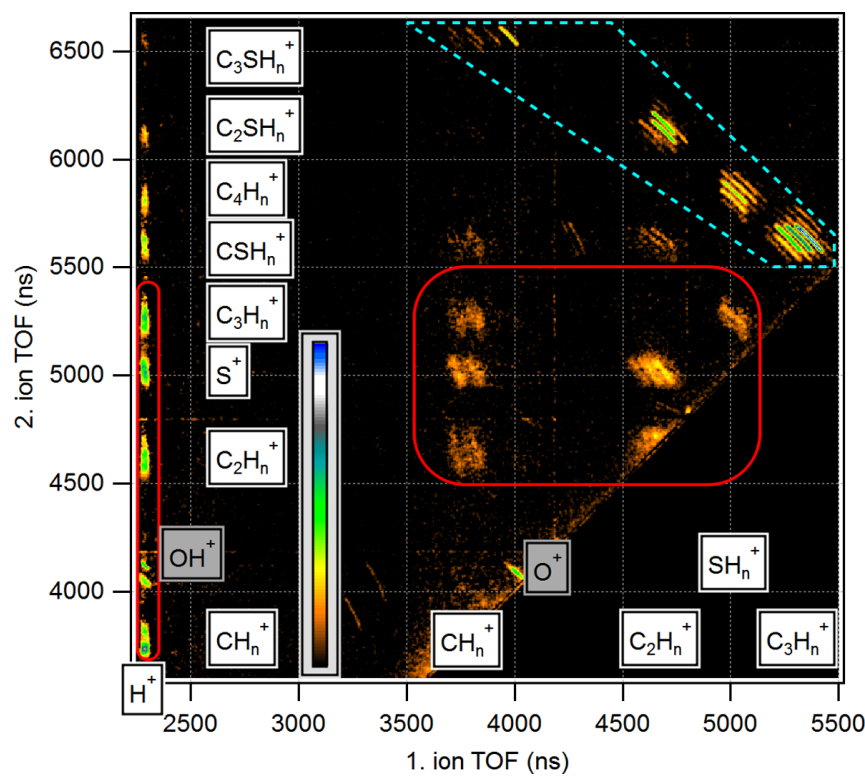

FIG. 3. Photoion-photoion coincidence (PIPICO) map of the dissociation of the thiophene dication following the $\mathrm{S} 2 p$ core ionization. The time-of-flight ranges of ionic species are indicated by the row and column of labels near the corresponding axis. The regions surrounded by the red line indicate the ion pairs for which strong pump-probe effects were observed. The region within the dashed blue line covers the two-body processes. 
FEL pulse against their flight times. The false-color scale represents the counts of ion pairs, and the islands appearing in the PIPICO map represent the various dissociation pathways. Thus the full PIPICO map is an experimental representation of the dissociation landscape of the thiophene dication. In constructing the PIPICO map of Fig. 3, the contribution from false coincidences (ion pairs formed by ions from different parent molecules) was subtracted. The ion TOFs depend not only on the ions' mass-to-charge ratio but also on the kineticenergy release (KER) in the dissociation process. Ion pairs with significant KER form patterns with negative slopes in the PIPICO map due to the momentum correlation between the two ions; such patterns are a reliable indication of true coincidence events. Some patterns, $\left(\mathrm{O}^{+}, \mathrm{O}^{+}\right),\left(\mathrm{H}^{+}, \mathrm{O}^{+}\right)$, and $\left(\mathrm{H}^{+}, \mathrm{OH}^{+}\right)$, are due to residual water or molecular oxygen ionization. All ions contributing to Fig. 3 are singly charged, except a weak pattern at around $\left(\mathrm{TOF}_{1} \approx 4300, \mathrm{TOF}_{2} \approx\right.$ 5600), where the first ion is $\mathrm{C}_{3} \mathrm{H}_{3}^{2+}$.

Figure 3 shows a very feature-rich dissociation landscape. For classification purposes, we will use the terms two-body and multibody dissociation, referring to the number of $\mathrm{C}$ - and S-containing ring fragments that are produced. The emission of hydrogen atoms or protons is referred to as $H$ loss, and it can accompany both the two-body and multibody processes or occur from a stable parent dication.

The intense, narrow patterns surrounded by the dashed blue line are due to two-body fragmentation, often accompanied by $\mathrm{H}$ loss and/or migration (creating the narrow stripes within the patterns). The central region of the PIPICO map encircled by the red line shows a number of more diffuse patterns that are due to a multibody fragmentation, also frequently accompanied by $\mathrm{H}$ loss or migration, such as

$$
\begin{aligned}
& \mathrm{C}_{4} \mathrm{SH}_{4}^{2+} \rightarrow \mathrm{C}_{2} \mathrm{H}_{n}^{0,+}+\mathrm{SH}_{m}^{0,+}+\mathrm{C}_{2} \mathrm{H}_{p}^{0,+}+k \mathrm{H}^{0,+}, \\
& \mathrm{C}_{4} \mathrm{SH}_{4}^{2+} \rightarrow \mathrm{C}_{3} \mathrm{H}_{n}^{0,+}+\mathrm{SH}_{m}^{0,+}+\mathrm{CH}_{p}^{0,+}+k \mathrm{H}^{0,+}, \\
& k=4-n-m-p .
\end{aligned}
$$

All combinations of the localization of the two positive charges on the right side of (1) are observable in Fig. 3. Ion pairs involving an ejected proton correspond to the encircled steeply sloped patterns on the left-hand side of the PIPICO map.

\section{B. Initial stages of dissociation as predicted by theory}

Although prior experiments [25,32] could not follow the early dynamics in the thiophene molecular dication, it could be investigated by theoretical self-consistent charge-densityfunctional tight-binding (SCC-DFTB) simulations, where the internal energy of the molecular dication was in the form of heat (the vibrational motion of atoms). In Ref. [25], a total of 12000 dissociation trajectories were simulated for internal energies ranging from 5.2 to $27 \mathrm{eV}$, and the individual trajectories at each temperature point were obtained by randomly varying the initial atomic velocity vectors at a set total kinetic energy.

Let us concentrate on the features of the simulations in the first few hundred femtoseconds. The calculations showed that fragmentation is almost universally preceded by a very fast opening of the ring and the formation of a transient linear

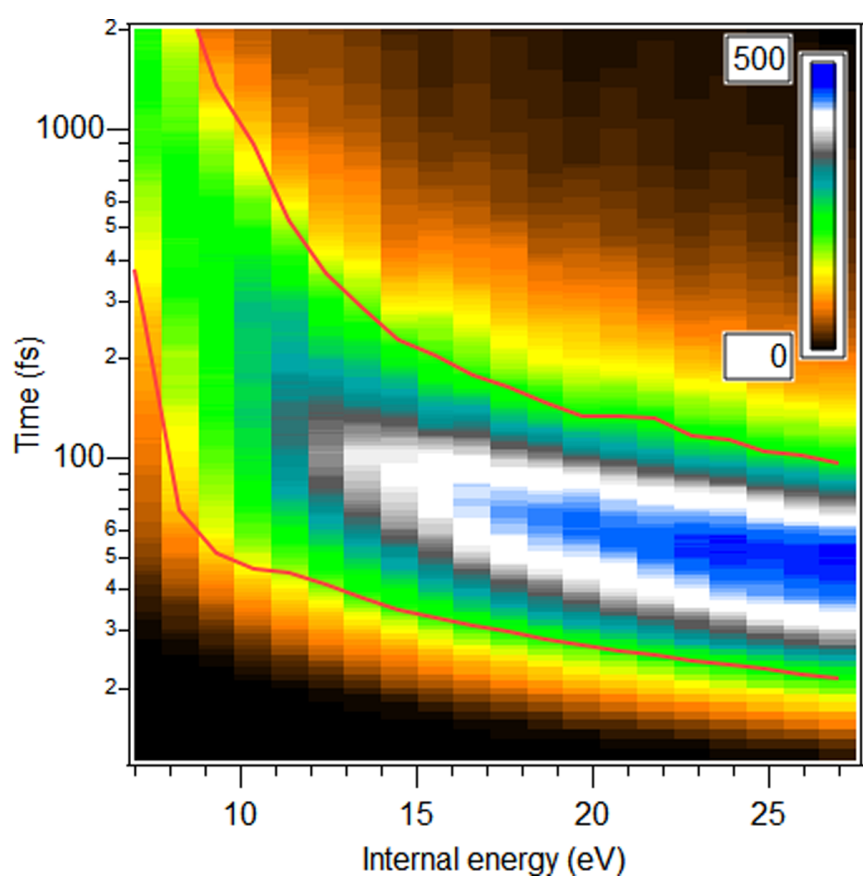

FIG. 4. Population of the transient linear geometry of the parent dication during molecular dynamics according to the SCC-DFTB simulations shown as a function of time and dependent on the internal energy of the molecule. Red curves mark the times of reaching the half-maximum population.

geometry of the parent dication. The location of the opening bond along the ring varies, with the highest (54-66\%) probability for the C-S bond to open. Once the ring opens, two-body or multibody fragmentation follows. Two example animations of such trajectories are given in the Supplemental Material [33]. The ring opening and fragmentation are often accompanied by $\mathrm{H}$ loss or migration.

Figure 4 shows how the transient linear geometry is created by ring opening and then depleted by dissociation, from a statistical analysis of 500 trajectories simulated for each internal vibrational energy value. Figure 4 thus represents the birth and survival of the transient geometry in the parent molecular dication. We can see a strong dependency of the relevant time constants (red curves) on the internal energy. In the present experiment, large internal energies of over $\approx 40 \mathrm{eV}$ in the molecular dication arise from populating highly excited final states in the course of the Auger decay [25,32].

In general, the SCC-DFTB simulations [25] match well with the observed dissociation landscape. The strongest individual dissociation pathway in both is

$$
\mathrm{C}_{4} \mathrm{SH}_{4}^{2+} \rightarrow \mathrm{CSH}^{+}+\mathrm{C}_{3} \mathrm{H}_{3}^{+} \text {. }
$$

The simulations predict all the observed two-body pathways (surrounded by dashed blue line area in Fig. 3) and also the numerous multibody fragmentation pathways, corresponding to the regions encircled by red lines in Fig. 3 (see also the animations in the Supplemental Material Ref. [33]) and single or multiple H-loss events. Only two-body events occur at the internal energies below $12 \mathrm{eV}$, while the multibody events become significant at higher energies. 

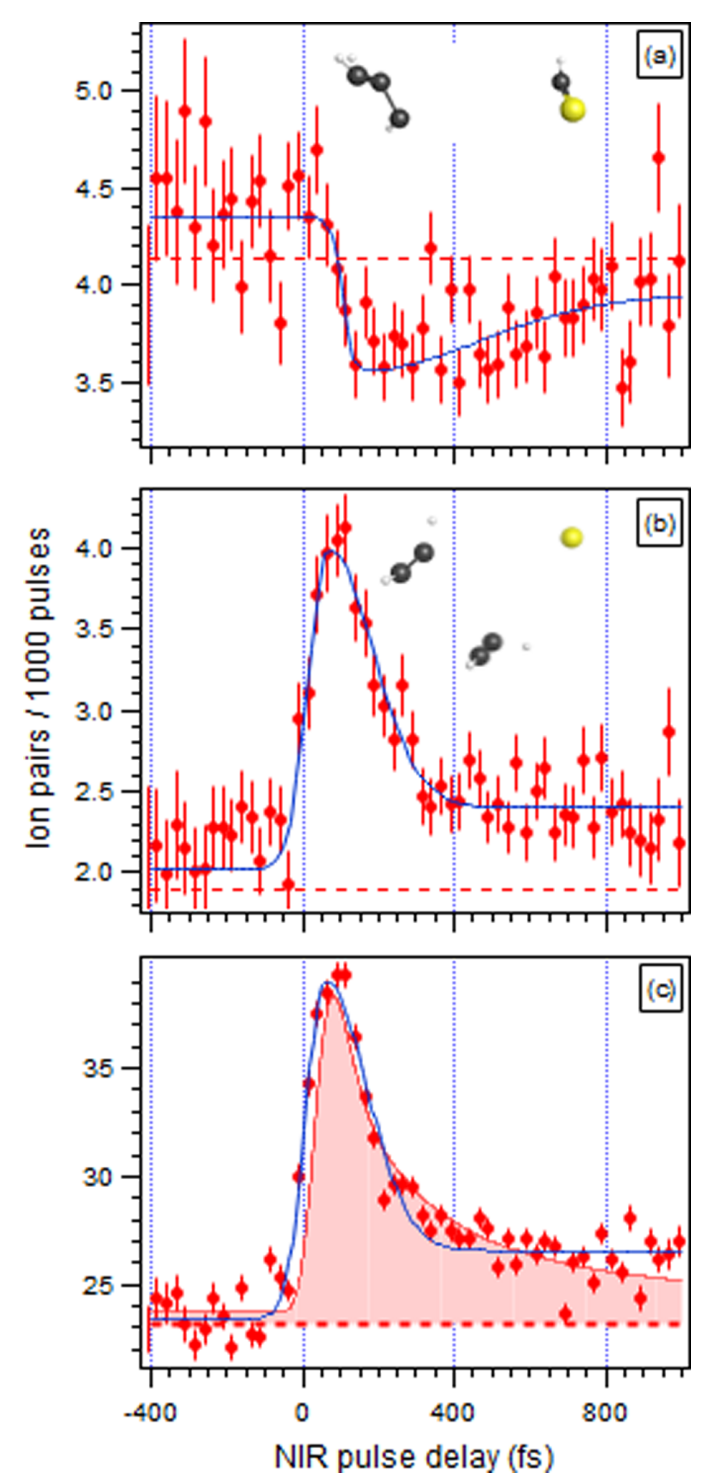

FIG. 5. (a) Measured ion-pair yield $\left(\mathrm{CSH}^{+}, \mathrm{C}_{3} \mathrm{H}_{3}^{+}\right)$from the strongest two-body pathway and (b) multibody fragmentation ionpair yield $\left(\mathrm{S}^{+}, \mathrm{C}_{2} \mathrm{H}_{n}^{+}\right)$, per 1000 pump-probe events. (c) Total multibody ion-pair yield from the areas encircled by the red lines in Fig. 3. Dashed lines are the yield values from a FEL-only measurement, and the blue curves are fits of the model function of (3) to the data. The shaded area in (c) shows the internal-energy-averaged population of the transient linear geometry from the SCC-DFTB simulations, convoluted by the temporal instrument function. Data error bars are based on Poisson statistics of ion-pair counting.

\section{Time-dependent effects of adding the NIR probe}

We begin the quantitative analysis of the structures already seen in the PIPICO map (Fig. 3) as ion-pair yields, adding the dimension of time delay between the FEL and the NIR pulses (the "NIR delay"; Fig. 5). In the experiment, the arrival time of the NIR pulse was scanned continuously across the FEL pulse, so that it in fact became the pump pulse at the negative delay values and the probe pulse at the positive ones. The data points of the yield curves in Fig. 5 were obtained by counting specific types of ion pairs formed in the pump-probe events.
The dashed lines indicate the ion-pair yields in the reference FEL-only measurement.

Figure 5(a) shows the ion-pair yield in the strongest individual two-body dissociation pathway (2), where one can see a clear drop of about $20 \%$ starting from a NIR delay of $\approx 110 \mathrm{fs}$. The effect is mostly transient with a slow recovery time of several hundred femtoseconds. From all two-body pathways seen in Fig. 3, only this pathway (2) shows statistically significant NIR-induced effects; overall the two-body processes are affected very little (see Appendix). Analyzing the yields of individual ions in this pathway narrows the effect down further as the depletion of the $\mathrm{C}_{3} \mathrm{H}_{3}^{+}$ion yield, while the $\mathrm{SCH}^{+}$population remains unaffected by NIR.

Figure 5(b) is an example of ion-pair yield $\left(\mathrm{CH}_{n}^{+}\right.$, $\mathrm{S}^{+}$) from a multibody pathway $\mathrm{C}_{4} \mathrm{SH}_{4}^{2+} \rightarrow \mathrm{C}_{2} \mathrm{H}_{n}^{+}+\mathrm{S}^{+}+$ $\mathrm{C}_{2} \mathrm{H}_{4-n}$. Here, a dramatically different behavior is seen: The yield more than doubles immediately when the NIR pulse arrives together with or after the FEL pulse. This strong transient enhancement is more short-lived than the negative effect in the two-body channel. Similar behavior, although of varying strength, is exhibited by all individual multibody ion-pair yields (see Appendix), and so we summarize our main experimental finding as a single curve in Fig. 5(c). It shows the multibody events corresponding to the ion pairs from the regions encircled by red in the PIPICO map in Fig. 3. Comparing the total multibody ion-pair yield [Fig. 5(c)] in the pump-probe experiment with the FEL-only yield confirms that an early NIR pulse (negative NIR delay) has no statistically significant effect. In the FEL-pump-NIR-probe region, one can identify a persistent component and a transient component of the enhancement. The ion, ion-pair, and ion-triplet yield curves were fitted using the following model function:

$$
Y(t)=Y_{0}+A_{p} \operatorname{erf}\left(\frac{t-t_{0}}{w}\right)+A_{t} \exp \left(\frac{-\left(t-t_{g}\right)^{2}}{\left(w+w_{t>t_{g}}\right)^{2}}\right)
$$

where the broadening parameter $w$ of the error function also defines the left-hand-side width of the asymmetric Gaussian. The half-maximum position of the Gaussian is aligned with the zero crossing of the error function by the term $t_{g}=t_{0}+$ $\sqrt{\ln 2} w$, creating a common rising edge, and the right-handside width of the Gaussian is modified by the additional broadening $w_{t>t_{g}}$. The intensity parameters are $Y_{0}, A_{p}$, and $A_{t}$. The FEL-delay scale was calibrated by the rising-edge position obtained from the fit of Fig. 5(c) (zeroing the model parameter $t_{0}$ ). All yield curves in Fig. 5 (see also additional yield curves in the Supplemental Material Ref. [33]) are given on this delay scale, regardless of their own fitted edge positions $t_{0}$ (see Table I). Using the $\mathrm{Xe}^{2+}$ yields from reference measurements for the delay scale calibration according to Ref. [34] is compatible with the above procedure within \pm 20 fs. Additional ion pair and triplet yields are given in the Appendix.

The blue curves in Fig. 5 show the fit of a model function representing both the persistent (error function) and the transient (asymmetric Gaussian) effects. The time constants from the fits of the multibody ion-pair yields in Fig. 5(b) and others are given in Table I.

From the fit of the ion-pair yield in Fig. 5(a), the NIR effect on the two-body process is delayed by $106(15) \mathrm{fs}$, but 
TABLE I. Edge positions and the edge and decay widths (in femtoseconds) of the transient NIR enhancements from least-squares fitting of ion-pair yields. Time delay $t_{0}=0$ is defined as the edge position in the fit of the combined multibody ion yield. The width parameters are according to Eq. (3) $\left(w_{\text {edge }}=w\right.$ and $w_{\text {decay }}=w+$ $\left.w_{t>t_{g}}\right)$.

\begin{tabular}{lccc}
\hline \hline Pair & \multicolumn{4}{c}{$t_{0}$} & $w_{\text {edge }}$ & $w_{\text {decay }}$ \\
\hline \multicolumn{4}{c}{ Multibody yields } \\
$\mathrm{CH}_{n}, \mathrm{CH}_{n}$ & $-7(15)$ & $63(35)$ & $222(91)$ \\
$\mathrm{CH}_{n}, \mathrm{C}_{2} \mathrm{H}_{n}$ & $-8(7)$ & $44(17)$ & $214(43)$ \\
$\mathrm{CH}_{n}, \mathrm{~S}$ & $11(7)$ & $60(16)$ & $173(36)$ \\
$\mathrm{CH}_{n}, \mathrm{C}_{3} \mathrm{H}_{n}$ & $9(10)$ & $27(33)$ & $241(78)$ \\
$\mathrm{C}_{2} \mathrm{H}_{n}, \mathrm{~S}$ & $9(9)$ & $71(21)$ & $128(44)$ \\
$\mathrm{S}_{2} \mathrm{C}_{3} \mathrm{H}_{n}$ & $-21(18)$ & $105(37)$ & $80(78)$ \\
$\mathrm{H}_{n}, \mathrm{CH}_{n}$ & $10(6)$ & $81(14)$ & $145(31)$ \\
$\mathrm{H}_{n}, \mathrm{C}_{2} \mathrm{H}_{n}$ & $-24(13)$ & $85(31)$ & $190(71)$ \\
$\mathrm{H}_{n}, \mathrm{~S}$ & $8(7)$ & $69(15)$ & $104(31)$ \\
$\mathrm{H}_{n}, \mathrm{C}_{3} \mathrm{H}_{n}$ & $-11(12)$ & $12(134)$ & $198(253)$ \\
$\mathrm{Combined}$ & $0.0(2.2)$ & $66(5)$ & $158(12)$ \\
$\mathrm{CSH}_{2} \mathrm{C}_{3} \mathrm{H}_{3}$ & \multicolumn{2}{c}{ Two-body yield } & \\
\hline \hline
\end{tabular}

then the falling-edge width of 42(30) fs can be fully ascribed to the temporal instrument function. The transient depletion partly recovers with the width of $455(100)$ fs. From the total multibody ion-pair yield in Fig. 5(c), we obtain a transient enhancement of 55\% over the FEL-only level and a persistent enhancement of $13 \%$. The broadening of the rising edge is $66(5) \mathrm{fs}$, and the decay width is $158(12) \mathrm{fs}$.

Comparing the pump-probe effects in the curves in Figs. 5(a) and 5(c), the first observation to make is that both the transient and persistent enhancements in the total multibody yields are much stronger than the decrease in the two-body yield. If the NIR effect were mainly enhanced dissociation due to the increase in the internal energy by nonionizing absorption, it would transfer intensity from the two-body yield to multibody yield, with mirroring behavior. This is not observed, leading to the conclusion that the NIR effect is primarily ionizing, transferring the post-Auger system evolving along any of the $M^{2+}$ potential-energy surfaces (Fig. 2) to the $\mathrm{M}^{3+}$ surfaces. The primary consequence of this is the increased number of ionized multibody fragments produced (at the expense of the neutral fragments in multibody processes), leading to a higher count of all possible multibody ion pairs. In the experiment, total ion-pair count from multibody processes increased by as much as $70 \%$ [Fig. 5(c)].

With the transient enhancements in ion-pair yields due to additional NIR ionization of the dicationic transient geometry, we expect changes also in the multibody kinematics, since an additional charged fragment would increase the momenta of individual ions in dissociation. The clearest experimental momentum data were obtained by investigating the $\mathrm{S}^{+}$ion, since it shows transient enhancements in all channels in which it appears and is accompanied by only a very low contribution of $\mathrm{SH}^{+}$ions, making its momentum determination the most accurate among the multibody fragments.

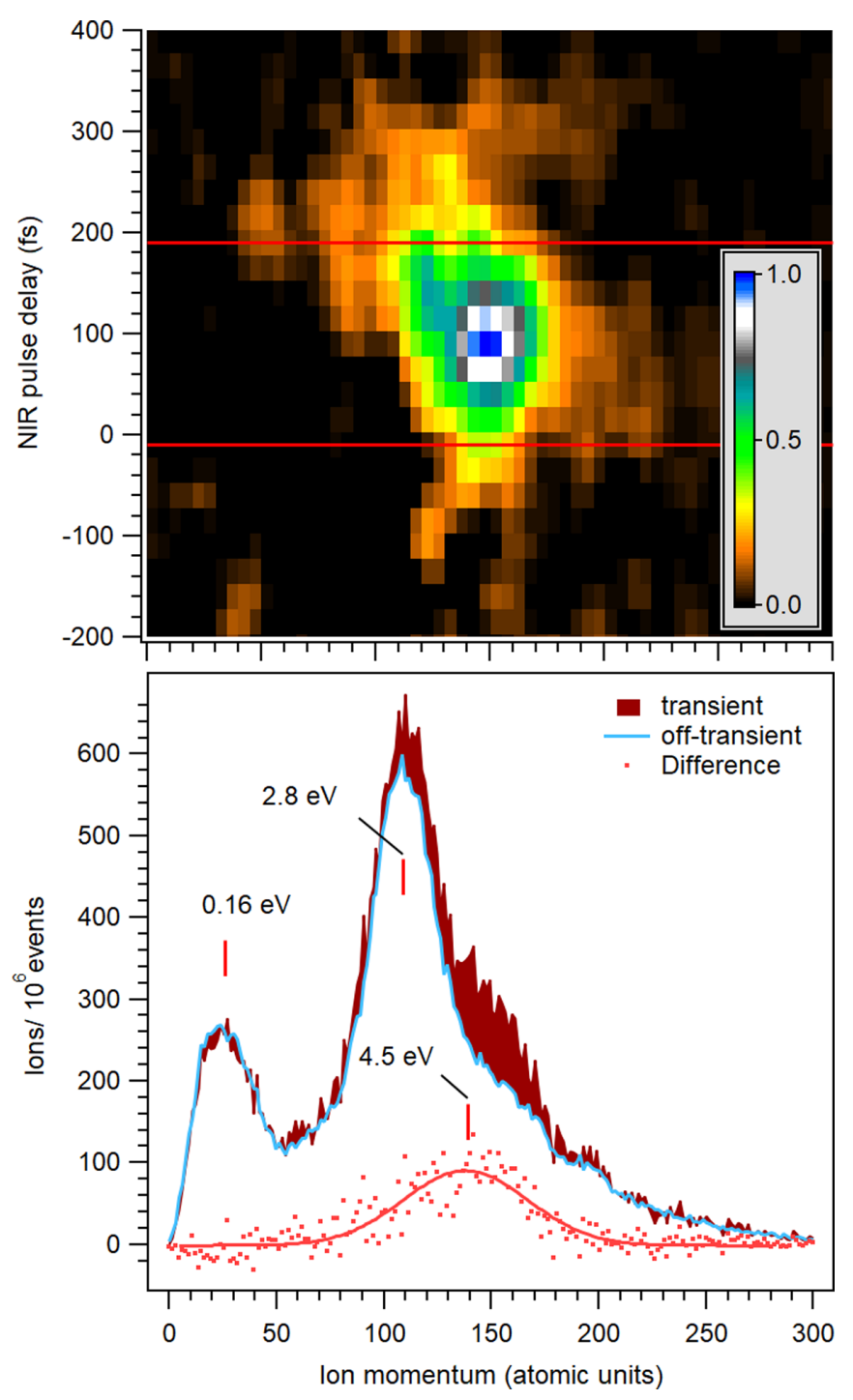

FIG. 6. Top: momentum distribution of $\mathrm{S}^{+}$ions as a function of the NIR delay, after subtracting the averaged NIR-early momentum distribution (NIR delay $<-300 \mathrm{fs}$ ). The color scale is set relative to the peak value of the distribution function over the shown range of ion momenta and pulse delays. Bottom: momentum distribution of the $\mathrm{S}^{+}$ions in the transient (between the red lines in the top panel) and off-transient regions of the NIR delay. Dots with a fitted Gaussian function are the difference of the two curves. The ion kinetic energies corresponding to the peak maxima are marked.

The top panel of Fig. 6 is a two-dimensional histogram of the momenta of all the $\mathrm{S}^{+}$ions detected in the pump-probe measurement, binned according to the NIR delays of the pump-probe events producing them. The histogram is given as a difference from the NIR-pump-FEL-probe momentum distribution, by subtracting the distribution curve of the $\mathrm{S}^{+}$ ions produced in the events where the NIR pulse arrives more than 300 fs before the FEL pulse. It is clearly seen that the ions from the NIR enhancement fall into a well-defined momentum range around 140 a.u., with a time profile of the transient enhancement region.

The bottom panel of Fig. 6 shows the $\mathrm{S}^{+}$ion's momentum distributions within and outside the transient NIR-delay range 


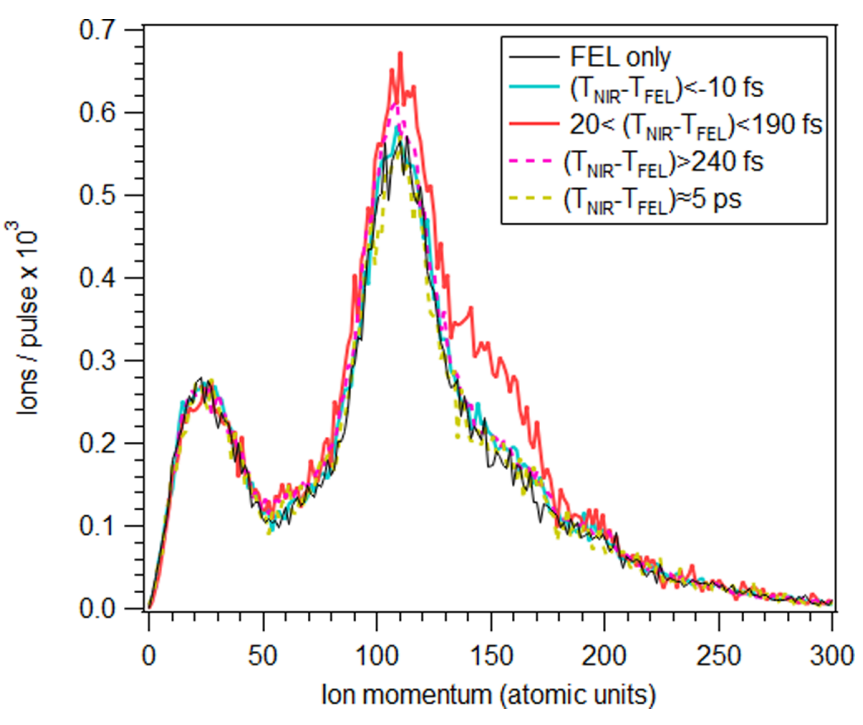

FIG. 7. Momentum distribution of $S^{+}$ions in various NIR-delay ranges and in the FEL-only measurement (black line). The range of the transient effects was set to the 20-to-190 fs FEL-NIR-pulse delay (red curve) and also the pre-transient (cyan curve), after-transient (dashed pink) and long FEL-delay (dashed yellow) ranges are shown.

and Fig. 7 gives a more detailed breakdown of the dependency of the momentum distribution on the pump-probe delay. Both momentum distribution curves in Fig. 6 show a first maximum corresponding to very small kinetic energy which is attributed to the residual $\mathrm{O}_{2}^{+}$contamination or a small amount of $\mathrm{S}^{+}$ fragments from direct valence ionization. The main maximum corresponds to $2.8 \mathrm{-eV}$ kinetic energy, while the ions from the transient enhancement have an average of $4.5-\mathrm{eV}$ kinetic energy.

Ion triplet yields provide even more dissociation-channelspecific information on multi-body fragmentation events, but suffer from lower statistics than the ion-pair yields.

We confirmed the assignment of the two maxima to the two- and three-ion pathways by a simple point-charge Coulomb explosion model [24,35], starting from the linear transient geometry and using the centers of mass of the fragments as starting coordinates. In a three-body concerted dicationic dissociation where, in addition to $\mathrm{S}^{+}$, only one $\mathrm{C}_{2} \mathrm{H}_{2}$ fragment obtains charge, the $\mathrm{S}^{+}$ion receives either 1.34 or $2.22 \mathrm{eV}$ of kinetic energy. If one considers also the possibility of a two-step process, first releasing the $\mathrm{S}^{+}$ions by $\mathrm{C}_{4} \mathrm{SH}_{4}^{2+} \rightarrow \mathrm{S}^{+}+\mathrm{C}_{4} \mathrm{H}_{4}^{+}$, then $\mathrm{S}^{+}$obtains $2.35 \mathrm{eV}$ of energy. In a concerted dissociation of a triply charged system with $\mathrm{S}^{+}$ and two $\mathrm{C}_{2} \mathrm{H}_{2}^{+}$ions, $\mathrm{S}^{+}$receives $4.19 \mathrm{eV}$ of kinetic energy (4.46 eV in the case of a two-step process $\mathrm{C}_{4} \mathrm{SH}_{4}^{2+} \rightarrow \mathrm{S}^{+}+$ $\mathrm{C}_{4} \mathrm{H}_{4}^{2+}$ ). These guideline values support our interpretation of the transient enhancement as due to quite a different process from the prevalent dicationic dissociation, namely, the more energetic breakup of a triply charged system.

\section{DISCUSSION}

\section{A. Probing the early dynamics}

At the starting point of molecular dynamics, the Auger decay populates numerous dicationic potential-energy sur- faces (PESs), two of which are shown schematically in Fig. 2, up to about $40 \mathrm{eV}$ above the double-ionization potential (DIP) [25]. Some of these states lie above the triple-ionization potential (TIP) and can therefore decay further onto the triply ionized PESs, leading to triple ion coincidences observable already in the FEL-only experiment (see Appendix for the ion-triplet yields). Consistently, the momentum distribution of the $\mathrm{S}^{+}$ions (Fig. 6, blue curve) has a tail in the region corresponding to the triply ionized dissociation even without the NIR-induced enhancement.

We concluded that the NIR probe facilitates the transfer of the system to the triply ionized PESs. First, we calculated the ionization potentials along one chosen dissociation trajectory from the SCC-DFTB simulations. The calculated TIP lies above the DIP by $23.3,19.9$, or $17.4 \mathrm{eV}$ in the ground-state, open-linear, and dissociated (with the $\mathrm{S}, \mathrm{C}_{2} \mathrm{H}_{2}$, and $\mathrm{C}_{2} \mathrm{H}_{2}$ fragments) geometries, respectively. (The edges of the bottom $\mathrm{M}^{2+}$ and the $\mathrm{M}^{3+}$ surfaces in Fig. 2 are from this calculation.) This energy is too high for efficient NIR ionization from $M^{2+}$ to $M^{3+}$ PESs. In the basic picture, Auger decay replaces the inner-shell vacancy with two vacancies in the outer- or innervalence molecular orbitals. Such a description of the Auger final states has been shown to be rather simplified, however, since due to the correlated motion of all valence electrons, an electron can transfer to an initially empty, Rydberg-like orbital [36]. These excited electrons have smaller binding energies than valence electrons, more easily overcome by the absorption of one or a few NIR photons. The combination of high-energy Auger final states and the presence of excited electrons due to electron correlation creates a suitable environment for efficient NIR ionization, such as observed in the laser-enabled Auger decay (LEAD) process [27,36,37]. It is worth emphasizing the key point of the NIR probing of the particular electron-correlated dicationic states involving an excited electron. The NIR probe acting on the pure two-hole states (in valence and inner-valence orbitals) would, consistent with the present experimental results, be quite insensitive to such a system, and the FEL-NIR pump-probe approach would not be a suitable one to study the dynamics in dicationic states that are prevalent after x-ray absorption in organic molecules and biomolecules. However, the electron correlation of the two-hole states with the states involving an excited Rydberg electron (three-hole, one-excited-electron states) makes the NIR probe sensitive to the dicationic states.

Such an environment arises very shortly after the core ionization, following the core-hole lifetime of a few femtoseconds, and creates the observed fast-rising edge of the transient NIR effects. The next questions are, What changes in the evolving systems cause these effects to fade, and what transient properties do we actually probe?

Let us first look at the early evolution of the molecular geometry. Figure 4 showed the population of the transient geometry of the parent dication as a function of time and internal energy, from the SCC-DFTB simulations. In Fig. 5(c), it is compared with the experimental total multibody ion-pair yield, after calculating a weighted average over the Auger final-state energies according to their theoretical density of states in the Auger spectrum [25]. The Auger final states 
with less than $10 \mathrm{eV}(10000 \mathrm{~K})$ internal energy were excluded, since the likelihood of NIR-induced third ionization from these states should be negligible. This particular cutoff energy was also chosen since neither the simulated trajectories nor the electron-energy-resolved experiment [25] show any multibody fragmentation channels below that energy. As the comparison shows, the temporal behavior of the transient linear parent geometry is quantitatively well matched with the NIR enhancement.

Turning to the changes in the electronic structure, one recognizes that the relaxation of the high-energy dicationic states towards the lowest-energy state can take place over time. This internal conversion transfers energy from electrons to the nuclear motion and is a fundamental mechanism of energy dissipation in organic molecules [2,38,39]. As the energy is transferred from the electronic to the nuclear subsystem, the initially excited dicationic electronic states are depleted, and the NIR-ionization probability by LEAD decreases accordingly. The internal conversion is not a process easily modeled and can occur in different systems over a broad time range [40]. Our observation of the decay width of the transient enhancement, about $160 \mathrm{fs}$, sets the lower limit to the internalconversion time constant.

It is of interest to consider briefly the relevant findings in other studies, although their different photoexcitation conditions (involving valence rather than the inner electron shells) do not allow for a direct comparison. Photoinduced thiophene ring opening has been observed following neutral excitations from the ground state to the $S_{1}$ state by Weinkauf et al. [1], who determined in a UV pump-probe experiment a time constant of $80 \mathrm{fs}$, associated with the ring opening by the cleavage of the $\mathrm{C}-\mathrm{S}$ bond (the prevalent cleavage site in our simulations also [25]). A subsequent theoretical study of the $S_{1}$ excited state in thiophene found that the subsequent dynamics of the open-ring structures is characterized by the interplay of internal conversion and intersystem crossing [41]. Other femtosecond pump-probe studies in the XUV energy range have drawn inferences about the timescale of the internal conversion in a dissociating molecular system. In a study by Månsson et al. [15], the authors observed transient effects in cationic states of thymidine. Their decay time constants of less than $200 \mathrm{fs}$ were interpreted as due to rapid decay via nonadiabatic coupling between electronic states. In another study of the radiosensitizer molecule 5-fluorouracil, both fast nonadiabatic-relaxation (30 fs) and slow (2.3 ps) internalconversion timescales were proposed as creating the transient pump-probe effects [42].

\section{B. Probing the late dynamics}

In the FEL-pump-late-NIR-probe region beyond the transient structures we observe weak persistent enhancements over the FEL-only baseline in multibody ion-pair yields, such as the plateau beyond $\approx 400$-fs delay in Fig. 5(c). The plateau continues to much longer FEL-NIR delays (was observed even at $5 \mathrm{ps}$ ) and therefore must be related to the interaction of the NIR pulse with the fragment species, produced by the FEL ionization. The most likely persistent NIR contribution to the multibody ion-pair yields comes from the ionization of neutral fragments, such as $\mathrm{C}_{1-3} \mathrm{H}_{1-4}$. The ionization energies of the neutral fragments are comparable to or even lower (starting from $6.6 \mathrm{eV}$ for the cyclopropenyl radical $\mathrm{C}_{3} \mathrm{H}_{3}$ [43]) than that of neutral thiophene, which is weakly ionized by the NIR pulse. Fundamentally, the persistent and transient NIR interactions are similar, but the former occurs in a nonevolving environment of stable neutral fragment species likely as multiphoton ionization.

The ion momentum distributions are also useful in probing the late dynamics. The $\mathrm{S}^{+}$ions showed a clear triple-ionization high-momentum transient enhancement. In contrast, the momentum distributions in the late persistent enhancement region are identical in shape to the pretransient region and also to the FEL-only distribution (more detailed ion momentum curves are given in the Appendix). The persistent component is seen as a slight increase in the ions with momenta corresponding to the doubly charged process. Although the NIR pulse eventually releases the third charge, the neutral fragments are ionized at larger separations where its Coulomb interaction with the two initially charged fragments is much weaker. Since the fragment separation at the time of the arrival of the NIR pulse is delay dependent, shorter pump-probe delays close to the transient region should give a larger change in the momenta of ions, and at long delays the change becomes negligible. Some tentative indications of such delay dependency of the momentum of the persistent enhancement are seen in the top panel of Fig. 6.

Lastly, we note that the NIR-probe effect on two-body channels can only be depletive. Generally, the two-body pathways are affected very little in our experiment. This is readily understood since the NIR pulse requires highly excited dicationic states for efficient ionization (depleting the two-body channels) but the two-body pathways are primarily associated with the low-energy dicationic states. The only two-body process exhibiting the NIR effects [Fig. 5(a)] exhibits a delayed onset of the transient NIR effect. The $\approx 100$-fs delay is well in line with the fragment separation time in the two-body process (2): A point-charge calculation using the center-of-mass positions of the two fragments in the ground-state geometry of thiophene as a starting point showed that in $100 \mathrm{fs}$ their separation increases from 1.9 to $6.4 \AA$-a reasonable distance to describe a fragmented system. The slow decay time of about 500 fs of the depleting NIR effect likely represents the timescale of the internal conversion of electronic energy within the $\mathrm{C}_{3} \mathrm{H}_{3}^{+}$fragment and/or geometry relaxation, slowly reducing the NIR pulse's ability to interact with it.

\section{SUMMARY}

We have demonstrated how the FEL-NIR pump-probe experiments shed light on the early period of the X-rayinduced molecular dissociation of organic molecules and can be suitable also for larger biomolecules. We suggest that the NIR enhancement is linked to the creation of highly excited electronic states by the Auger decay of the coreionized molecules, as a particular feature of x-ray ionization. These excited states likely involve electrons promoted to 
Rydberg-type orbitals, allowing the NIR probe to interact efficiently. However, this interaction is transient since the highly excited states are continually depleted by internal conversion, transferring energy to vibrational motion. Exactly how the electronic structure changes and accommodates to the changing geometry has a direct bearing on the ability of the NIR probe to interact with the system.

The observations on our sample system, thiophene, in combination with theoretical modeling led us to conclude that the cyclic parent thiophene dication quickly opens up into a linear geometry, which then dissociates. Calculations suggest that the dissociation has a certain latency, since that linear geometry can occasionally survive for tens, even hundreds, of femtoseconds, even at high internal energies. We suggest that it is this open-ring parent geometry that ibeing probed and that the time structure of the transient NIR-induced enhancements reflects the statistical survival probability (lifetime) of this transient geometry. Eventually, the NIR effect fades into much weaker, persistent interaction with the stable fragment species. Perhaps surprisingly, our study also leads to the conclusion that the decay of the energetic electronic states by internal conversion is slow enough, over $\approx 160 \mathrm{fs}$, to allow for significant concurrent nuclear dynamics.

The prominence of electronic relaxation and geometry changes in the early photodynamics underscores the importance of nonadiabatic couplings in this period. Both the survival of the parent dication even at strongly unfavorable conditions and the redistribution of the internal energy over time are properties that have relevance well beyond the technicalities of pump-probe experiments. The first might significantly affect the final extent and nature of the radiation damage as fragments are released over time by the primary and secondary (electron and fragment collisions) ionizations. The second affects our ability to accurately model the dissociation landscape in larger quantum systems.

New time-resolved experimental opportunities offered by the FEL facilities with very high pulse repetition rates will certainly reveal new details of the formative periods of the photodissociation landscapes of molecules. Here, we demonstrated how, in the FEL-NIR pump-probe scheme, the NIR probe becomes sensitive to transient excited dicationic states of the molecule, created by the Auger relaxation of corehole states. We showed the feasibility of this experimental approach in the example of a medium-sized organic molecule. This technique would likely also be beneficial, for example, in time-tracing the early evolution of $x$-ray radiation damage in the molecular building blocks of much larger biomolecules.

Using pump-probe schemes such as the one in this paper and adding selectivity to the internal energy by also recording the Auger electrons' energies will help in investigating the parent molecule's survival times and the fast energy conversions following the ionization event.

\section{ACKNOWLEDGMENTS}

This study was supported by the X-ray Free Electron Laser Utilization Research Project and the X-ray Free Electron Laser Priority Strategy Program of the Ministry of Education, Culture, Sports, Science and Technology of Japan (MEXT), by the Proposal Program of SACLA Experimental Instruments of RIKEN, by the research program "Dynamic Alliance for Open Innovation Bridging Human, Environment and Materials" at the Network Joint Research Center for Materials and Devices, by the IMRAM project, and by the Japan Society for the Promotion of Science (JSPS). E.K., J.N., and E.P. acknowledge financial support from the Academy of Finland, and D.Y. was supported by a grant-in-aid from Tohoku University Institute for Promoting Graduate Degree Programs, Division for Interdisciplinary Advanced Research and Education. J.P. and P.J. acknowledge support from the Swedish Research Council and the Swedish Foundation for Strategic Research. We thank the staff of the SACLA facility for their help in preparation of and during the experiment.

\section{APPENDIX: ION-PAIR AND ION-TRIPLET YIELDS}

In this appendix, further details on the ion-pair and iontriplet yields as a function of time are presented. Firstly, Fig. 8 shows the total ion-pair yield from all detected two-body dissociation channels.

Figure 9 gives ion-pair yields from eight observed strong multibody dissociation channels within the circled regions of Fig. 3.

Ion-triplet yields provide even more dissociation-channelspecific information on multibody fragmentation events but suffer from lower statistics than the ion-pair yields. Some examples are shown in Fig. 10.

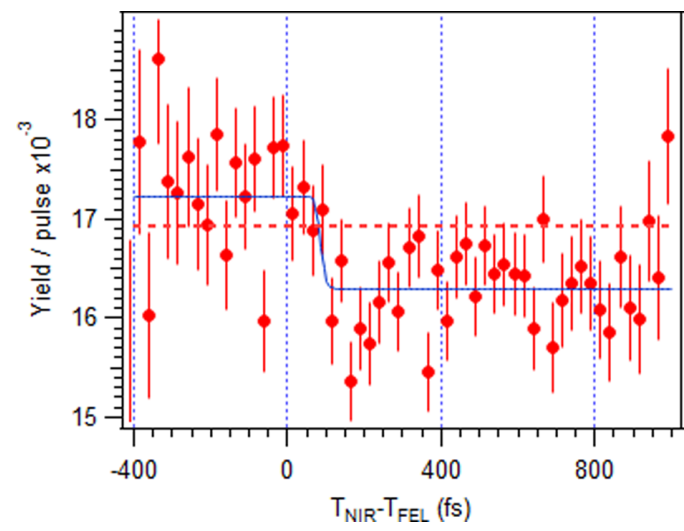

FIG. 8. Combined ion-pair yield of all two-body channels, as a function of NIR-delay. Dashed line is the yield value from a FELonly measurement. 

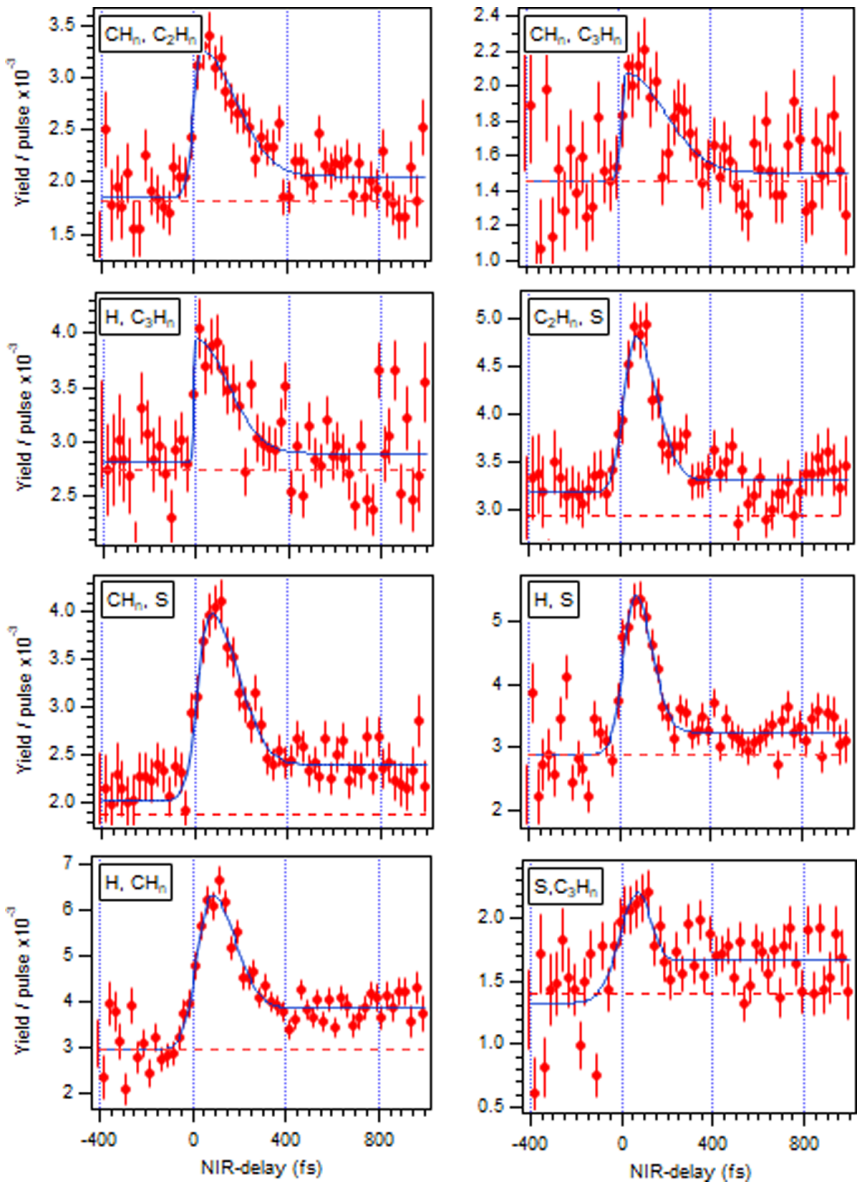

FIG. 9. Ion-pair yields of multi-body fragmentation events, as a function of NIR-delay. Dashed lines are yield values from a FELonly measurement.
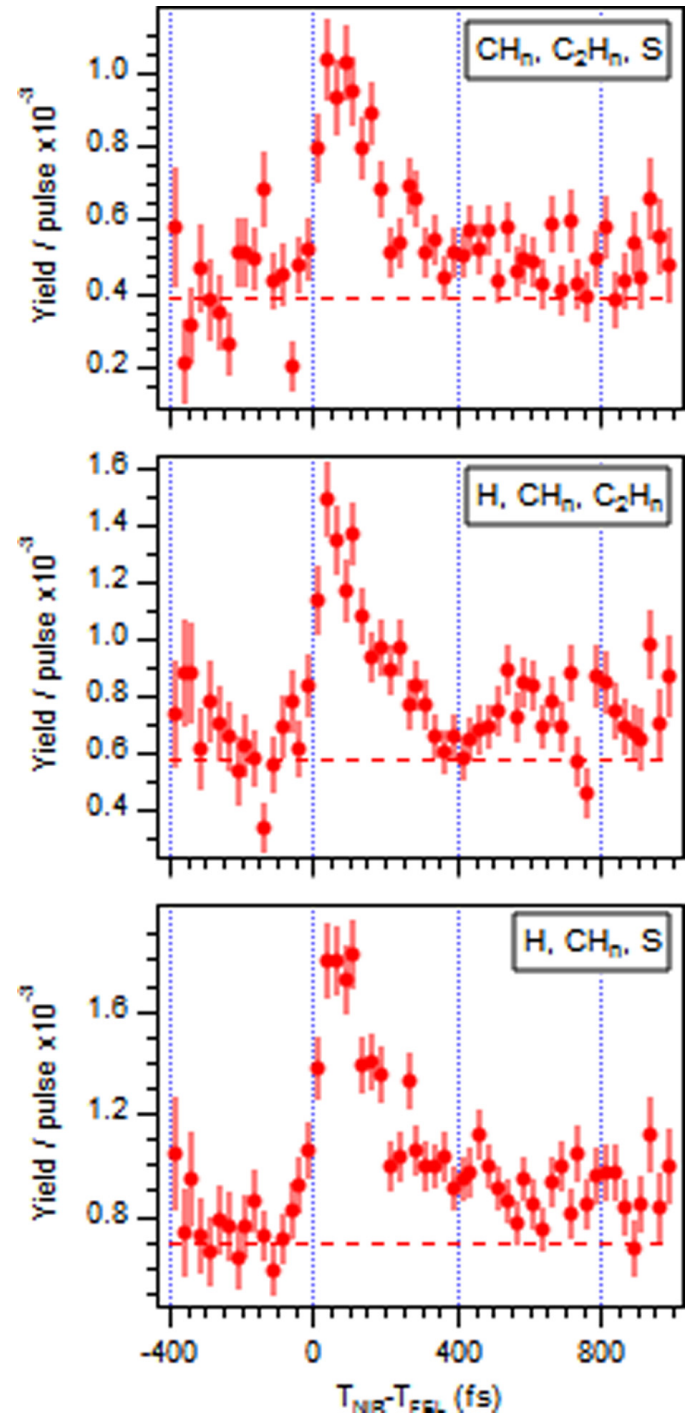

FIG. 10. Ion triplet yields of some prominent multi-body channels, as a function of NIR-delays. Dashed lines are the yield values from a FEL-only measurement.
[1] R. Weinkauf, L. Lehr, E. W. Schlag, S. Salzmann, and C. M. Marian, Ultrafast dynamics in thiophene investigated by femtosecond pump probe photoelectron spectroscopy and theory, Phys. Chem. Chem. Phys. 10, 393 (2008).

[2] T. J. A. Wolf, R. H. Myhre, J. P. Cryan, S. Coriani, R. J. Squibb, A. Battistoni, N. Berrah, C. Bostedt, P. Bucksbaum, G. Coslovich, R. Feifel, K. J. Gaffney, J. Grilj, T. J. Martinez, S. Miyabe, S. P. Moeller, M. Mucke, A. Natan, R. Obaid, T. Osipov et al., Probing ultrafast $\pi \pi * / n \pi *$ internal conversion in organic chromophores via K-edge resonant absorption, Nat. Commun. 8, 29 (2017).

[3] Handbook of Thiophene-Based Materials, edited by I. F. Perepichka and D. F. Perepichka (Wiley, Chichester, UK, 2009).

[4] I. Luzon, E. Livshits, K. Gope, R. Baer, and D. Strasser, Making sense of Coulomb explosion imaging, J. Phys. Chem. Lett. 10, 1361 (2019).
[5] S. Scheit, S. Goswami, H.-D. Meyer, and H. Köppel, Fully quantal treatment of nonadiabatic molecular photodynamics: General considerations and application to the benzene cation, Comput. Theor. Chem. 1150, 71 (2019).

[6] S. Hsieh and J. H. Eland, Reaction dynamics of three-body dissociations in triatomic molecules from single-photon double ionization studied by a time- and position-sensitive coincidence method, J. Phys. B: At., Mol. Opt. Phys. 30, 4515 (1997).

[7] R. J. Squibb, M. Sapunar, A. Ponzi, R. Richter, A. Kivimäki, O. Plekan, P. Finetti, N. Sisourat, V. Zhaunerchyk, T. Marchenko, L. Journel, R. Guillemin, R. Cucini, M. Coreno, C. Grazioli, M. Di Fraia, C. Callegari, K. C. Prince, P. Decleva, M. Simon et al., Acetylacetone photodynamics at a seeded free-electron laser, Nat. Commun. 9, 63 (2018). 
[8] J. Ullrich, A. Rudenko, and R. Moshammer, Free-electron lasers: New avenues in molecular physics and photochemistry, Annu. Rev. Phys. Chem. 63, 635 (2012).

[9] L. Young, K. Ueda, M. Gühr, P. H. Bucksbaum, M. Simon, S. Mukamel, N. Rohringer, K. C. Prince, C. Masciovecchio, M. Meyer, A. Rudenko, D. Rolles, C. Bostedt, M. Fuchs, D. A. Reis, R. Santra, H. Kapteyn, M. Murnane, H. Ibrahim, F. Légaré et al., Roadmap of ultrafast x-ray atomic and molecular physics, J. Phys. B: At., Mol. Opt. Phys. 51, 032003 (2018).

[10] K. Ueda, E. Sokell, S. Schippers, F. Aumayr, H. Sadeghpour, J. Burgdörfer, C. Lemell, X.-M. Tong, T. Pfeifer, F. Calegari, A. Palacios, F. Martin, P. Corkum, G. Sansone, E. V. Gryzlova, A. N. Grum-Grzhimailo, M. N. Piancastelli, P. M. Weber, T. Steinle, K. Amini et al., Roadmap on photonic, electronic and atomic collision physics: I. Light-matter interaction, J. Phys. B: At., Mol. Opt. Phys. 52, 171001 (2019).

[11] J. van Tilborg, T. K. Allison, T. W. Wright, M. P. Hertlein, R. W. Falcone, Y. Liu, H. Merdji, and A. Belkacem, Femtosecond isomerization dynamics in the ethylene cation measured in an EUV-pump NIR-probe configuration, J. Phys. B: At., Mol. Opt. Phys. 42, 081002 (2009).

[12] Y. H. Jiang, A. Rudenko, O. Herrwerth, L. Foucar, M. Kurka, K. U. Kühnel, M. F. Lezius, M. Kling, J. van Tilborg, A. Belkacem, K. Ueda, S. Düsterer, R. Treusch, C. D. Schröter, R. Moshammer, and J. Ullrich, Ultrafast Extreme Ultraviolet Induced Isomerization of Acetylene Cations, Phys. Rev. Lett. 105, 263002 (2010)

[13] T. K. Allison, H. Tao, W. J. Glover, T. W. Wright, A. M. Stooke, C. Khurmi, J. van Tilborg, Y. Liu, R. W. Falcone, T. J. Martínez, and A. Belkacem, Ultrafast internal conversion in ethylene. II. Mechanisms and pathways for quenching and hydrogen elimination, J. Chem. Phys. 136, 124317 (2012).

[14] N. Hartmann, S. Bhattacharyya, F. Schlaepfer, M. Volkov, Z. Schumacher, M. Lucchini, L. Gallmann, U. Rothlisberger, and U. Keller, Ultrafast nuclear dynamics of the acetylene cation $\mathrm{C}_{2} \mathrm{H}_{2}^{+}$and its impact on the infrared probe pulse induced $\mathrm{C}-\mathrm{H}$ bond breaking efficiency, Phys. Chem. Chem. Phys. 21, 18380 (2019).

[15] E. P. Mänsson, S. De Camillis, M. C. Castrovilli, M. Galli, M. Nisoli, F. Calegari, and J. B. Greenwood, Ultrafast dynamics in the DNA building blocks thymidine and thymine initiated by ionizing radiation, Phys. Chem. Chem. Phys. 19, 19815 (2017).

[16] B. F. Murphy, L. Fang, M.-H. Chen, J. D. Bozek, E. Kukk, E. P. Kanter, M. Messerschmidt, T. Osipov, and N. Berrah, Multiphoton $L$-shell ionization of $\mathrm{H}_{2} \mathrm{~S}$ using intense $\mathrm{x}$-ray pulses from a free-electron laser, Phys. Rev. A 86, 053423 (2012).

[17] O. Kornilov, M. Eckstein, M. Rosenblatt, C. P. Schulz, K. Motomura, A. Rouzée, J. Klei, L. Foucar, M. Siano, A. Lübcke, F. Schapper, P. Johnsson, D. M. P. Holland, T. Schlathölter, T. Marchenko, S. Düsterer, K. Ueda, M. J. J. Vrakking, and L. J. Frasinski, Coulomb explosion of diatomic molecules in intense XUV fields mapped by partial covariance, J. Phys. B: At., Mol. Opt. Phys. 46, 164028 (2013).

[18] B. Erk, D. Rolles, L. Foucar, B. Rudek, S. Epp, M. Cryle, C. Bostedt, S. Schorb, J. Bozek, A. Rouzee, A. Hundertmark, T. Marchenko, M. Simon, F. Filsinger, L. Christensen, S. De, S. Trippel, J. Küpper, H. Stapelfeldt, S. Wada et al., Ultrafast Charge Rearrangement and Nuclear Dynamics upon Inner-Shell
Multiple Ionization of Small Polyatomic Molecules, Phys. Rev. Lett. 110, 053003 (2013).

[19] B. Erk, R. Boll, S. Trippel, D. Anielski, L. Foucar, B. Rudek, S. W. Epp, R. Coffee, S. Carron, S. Schorb, K. R. Ferguson, M. Swiggers, J. D. Bozek, M. Simon, T. Marchenko, J. Kupper, I. Schlichting, J. Ullrich, C. Bostedt, D. Rolles et al., Imaging charge transfer in iodomethane upon x-ray photoabsorption, Science 345, 288 (2014).

[20] B. Rudek, S.-K. Son, L. Foucar, S. W. Epp, B. Erk, R. Hartmann, M. Adolph, R. Andritschke, A. Aquila, N. Berrah, C. Bostedt, J. Bozek, N. Coppola, F. Filsinger, H. Gorke, T. Gorkhover, H. Graafsma, L. Gumprecht, A. Hartmann, G. Hauser et al., Ultra-efficient ionization of heavy atoms by intense X-ray free-electron laser pulses, Nat. Photonics 6, 858 (2012).

[21] A. Rudenko, L. Inhester, K. Hanasaki, X. Li, S. J. Robatjazi, B. Erk, R. Boll, K. Toyota, Y. Hao, O. Vendrell, C. Bomme, E. Savelyev, B. Rudek, L. Foucar, S. H. Southworth, C. S. Lehmann, B. Kraessig, T. Marchenko, M. Simon, K. Ueda et al., Femtosecond response of polyatomic molecules to ultra-intense hard X-rays, Nature (London) 546, 129 (2017).

[22] K. Motomura, E. Kukk, H. Fukuzawa, S.-i. Wada, K. Nagaya, S. Ohmura, S. Mondal, T. Tachibana, Y. Ito, R. Koga, T. Sakai, K. Matsunami, A. Rudenko, C. Nicolas, X.-J. Liu, C. Miron, Y. Zhang, Y. Jiang, J. Chen, M. Anand et al., Charge and nuclear dynamics induced by deep inner-shell multiphoton ionization of $\mathrm{CH}_{3} \mathrm{I}$ molecules by intense x-ray free-electron laser pulses, J. Phys. Chem. Lett. 6, 2944 (2015).

[23] K. Nagaya, K. Motomura, E. Kukk, Y. Takahashi, K. Yamazaki, S. Ohmura, H. Fukuzawa, S. Wada, S. Mondal, T. Tachibana, Y. Ito, R. Koga, T. Sakai, K. Matsunami, K. Nakamura, M. Kanno, A. Rudenko, C. Nicolas, X.-J. Liu, C. Miron et al., Femtosecond charge and molecular dynamics of I-containing organic molecules induced by intense X-ray free-electron laser pulses, Faraday Discuss. 194, 537 (2016).

[24] E. Kukk, H. Myllynen, K. Nagaya, S. Wada, J. D. Bozek, T. Takanashi, D. You, A. Niozu, K. Kooser, T. Gaumnitz, E. Pelimanni, M. Berholts, S. Granroth, N. Yokono, H. Fukuzawa, C. Miron, and K. Ueda, Coulomb implosion of tetrabromothiophene observed under multiphoton ionization by free-electron-laser soft-x-ray pulses, Phys. Rev. A 99, 023411 (2019).

[25] E. Kukk, D. T. Ha, Y. Wang, D. G. Piekarski, S. Diaz-Tendero, K. Kooser, E. Itälä, H. Levola, M. Alcamí, E. Rachlew, and F. Martín, Internal energy dependence in x-ray-induced molecular fragmentation: An experimental and theoretical study of thiophene, Phys. Rev. A 91, 043417 (2015).

[26] M. S. Schuurman and A. Stolow, Dynamics at conical intersections, Annu. Rev. Phys. Chem. 69, 427 (2018).

[27] D. You, K. Ueda, M. Ruberti, K. L. Ishikawa, P. A. Carpeggiani, T. Csizmadia, L. G. Oldal, H. N G, G. Sansone, P. K. Maroju, K. Kooser, C. Callegari, M. D. Fraia, O. Plekan, L. Giannessi, E. Allaria, G. D. Ninno, M. Trovó, L. Badano, B. Diviacco et al., A detailed investigation of single-photon laser enabled Auger decay in neon, New J. Phys. 21, 113036 (2019).

[28] S. Owada, K. Togawa, T. Inagaki, T. Hara, T. Tanaka, Y. Joti, T. Koyama, K. Nakajima, H. Ohashi, Y. Senba, T. Togashi, K. Tono, M. Yamaga, H. Yumoto, M. Yabashi, H. Tanaka, and T. Ishikawa, A soft X-ray free-electron laser beamline at SACLA: 
The light source, photon beamline and experimental station, J. Synchrotron Radiat. 25, 282 (2018).

[29] M. Yabashi, H. Tanaka, and T. Ishikawa, Overview of the SACLA facility, J. Synchrotron Radiat. 22, 477 (2015).

[30] S. Owada, K. Nakajima, T. Togashi, T. Katayama, H. Yumoto, H. Ohashi, and M. Yabashi, Arrival timing diagnostics at a soft X-ray free-electron laser beamline of SACLA BL1, J. Synchrotron Radiat. 26, 887 (2019).

[31] H. Fukuzawa, K. Nagaya, and K. Ueda, Advances in instrumentation for gas-phase spectroscopy and diffraction with short-wavelength free electron lasers, Nucl. Instrum. Methods Phys. Res., Sect. A 907, 116 (2018).

[32] R. G. Hayes and W. Eberhardt, Electron-ion coincidences studies of the fragmentation of thiophene and of tetrahydrothiophene upon core ionization, J. Chem. Phys. 94, 397 (1991).

[33] See Supplemental Material at http://link.aps.org/supplemental/ 10.1103/PhysRevResearch.3.013221 for example animations of the ring opening and fragmentation.

[34] D. Rolles, R. Boll, B. Erk, D. Rompotis, and B. Manschwetus, An experimental protocol for femtosecond NIR/UV - XUV pump-probe experiments with free-electron lasers, J. Visualized Exp. 140, e57055 (2018).

[35] E. Kukk, K. Motomura, H. Fukuzawa, K. Nagaya, and K. Ueda, Molecular dynamics of XFEL-induced photo-dissociation, revealed by ion-ion coincidence measurements, Appl. Sci. 7, 531 (2017).

[36] B. Cooper and V. Averbukh, Single-Photon Laser-Enabled Auger Spectroscopy for Measuring Attosecond Electron-Hole Dynamics, Phys. Rev. Lett. 111, 083004 (2013).
[37] D. Iablonskyi, K. Ueda, K. L. Ishikawa, A. S. Kheifets, P. Carpeggiani, M. Reduzzi, H. Ahmadi, A. Comby, G. Sansone, T. Csizmadia, S. Kuehn, E. Ovcharenko, T. Mazza, M. Meyer, A. Fischer, C. Callegari, O. Plekan, P. Finetti, E. Allaria, E. Ferrari et al., Observation and Control of Laser-Enabled Auger Decay, Phys. Rev. Lett. 119, 073203 (2017).

[38] M. Fagnoni, Modern Molecular Photochemistry of Organic Molecules. Von Nicholas J. Turro, V. Ramamurthy und Juan C. Scaiano, Angew. Chem. 122, 6859 (2010).

[39] R. R. Valiev, V. N. Cherepanov, G. V. Baryshnikov, and D. Sundholm, First-principles method for calculating the rate constants of internal-conversion and intersystemcrossing transitions, Phys. Chem. Chem. Phys. 20, 6121 (2018).

[40] H. Kuhn, H.-D. Försterling, and D. H. Waldeck, Principles of Physical Chemistry, 2nd ed. (Wiley, Hoboken, NJ, 2009).

[41] T. Schnappinger, P. Kölle, M. Marazzi, A. Monari, L. González, and R. de Vivie-Riedle, Ab initio molecular dynamics of thiophene: The interplay of internal conversion and intersystem crossing, Phys. Chem. Chem. Phys. 19, 25662 (2017).

[42] P. Çarçabal, D. Descamps, S. Petit, Y. Mairesse, V. Blanchet, and R. Cireasa, Using high harmonic radiation to reveal the ultrafast dynamics of radiosensitiser molecules, Faraday Discuss. 194, 407 (2016).

[43] NIST Chemistry WebBook: NIST Standard Reference Database Number 69, edited by P. J. Linstrom and W. G. Mallard (National Insitute of Standards and Technology, Gaithersburg, MD, 2000). 